\title{
On the Influence of Extracellular
}

\section{Fluid Volume Expansion on}

\section{Bicarbonate Reabsorption in the Rat}

\author{
Mabel L. Purkerson, Herbert Lubowitz, Rose W. White, and \\ NeAL S. Bricker \\ From the Renal Division, Department of Internal Medicine, Washington \\ University School of Medicine, St. Louis, Missouri 63110
}

\begin{abstract}
A B S T RAC T Bicarbonate reabsorption is classically regarded as a rate-limited process characterized by saturation kinetics. The tubular maximum ( $\mathrm{Tm}$ ), however, varies with glomerular filtration rate. Thus bicarbonate reabsorption, in common with sodium reabsorption, is characterized by glomerulo-tubular balance. The examination of bicarbonate reabsorption is accomplished using the bicarbonate titration technique; however, this method in its traditional form leads to marked expansion of extracellular fluid (ECF) volume. The possibility exists, therefore, that glomerulo-tubular balance for bicarbonate is altered by the volume expansion and thus that the classic pattern of reabsorption may actually reflect inhibited bicarbonate reabsorptive capacity. The present studies were performed in rats to examine this possibility. Bicarbonate titration studies were performed in two groups of animals: (a) those in which ECF volume expansion was minimized; and $(b)$ those in which ECF volume expansion was exaggerated. In the first group, no $\mathrm{Tm}$ for bicarbonate was observed either in the majority of individual rats studied or in a group plot for all rats studied despite the fact that plasma bicarbonate concentrations were increased to values in excess of $60 \mathrm{mEq} /$ liter. In the second group, a clear Tm was demonstrated both in individual animals and in group data and there was a lowered threshold for the excretion of bicarbonate. The data thus lend support to the view that the "normal" $\mathrm{Tm}$ for bicarbonate may actually represent an inhibited level of bicarbonate reabsorption induced by ECF volume expansion.
\end{abstract}

This work was presented in part at the Annual Meeting of the American Federation for Clinical Research, Atlantic City, N. J., 30 April 1967.

Dr. N. S. Bricker is a recipient of a U. S. Public Health Service Research Career Award.

Received for publication 24 January 1969 and in revised form 3 April 1969.

\section{INTRODUCTION}

The reabsorption of bicarbonate by the mammalian kidney is believed to be characterized by saturation kinetics $(1,2)$. The relationship between the apparent maximum velocity of transport and the substrate (i.e. bicarbonate) concentration of the glomerular filtrate is not a simple one however, in that the tubular maximum ( $\mathrm{Tm}$ ) for bicarbonate varies with glomerular filtration rate (GFR). Thus, under experimental conditions wherein a $\mathrm{Tm}$ is demonstrable, there appears to exist a form of glomerulo-tubular balance for bicarbonate which is analogous to glomerulo-tubular balance for sodium. To delineate the normal pattern for bicarbonate reabsorption, the bicarbonate titration technique has been employed. However, this procedure in its traditional form involves substantial expansion of extracellular fluid (ECF) volume. Since expansion of extracellular fluid volume has been shown to have profound effects on proximal tubular sodium reabsorption, and indeed to reset glomerulo-tubular balance for sodium, the possibility exists that the accepted "normal" pattern for bicarbonate reabsorption may be influenced by the experimental method. Accordingly, the present studies were undertaken to reexamine bicarbonate reabsorption using a bicarbonate titration technique which minimized extracellular fluid volume expansion. Studies also were performed during exaggerated ECF volume expansion.

\section{METHODS}

Experiments were performed on unanesthetized female Sprague-Dawley rats weighing between 225 and $250 \mathrm{~g}$. Two types of titration experiments were performed. The first was designed to restrict the expansion of ECF volume to the minimal level consistent with obtaining the appropriate stepwise elevation of plasma bicarbonate concentrations. In the second type of titration experiment ECF expansion was exaggerated. 
The preparation of the animals for study was accomplished in the manner described in detail previously (3). The rats were anesthetized lightly with ether to allow for the insertion of arterial, venous, and bladder catheters. After completion of the surgical procedures, the anesthesia was discontinued and a period of $1 \frac{1}{2}-2 \mathrm{hr}$ was allowed for the animals to recover completely from the anesthetic. Urine was collected through a soft silastic catheter (o.D. $1.25 \mathrm{~mm}$ ).

All of the sustaining infusions contained a sodium concentration of $140 \mathrm{mEq} /$ liter. The rate of bicarbonate administration was adjusted by increasing the bicarbonate concentration of the infusate progressively from 0 (in the control periods)

TABLE I

Representative Bicarbonate Titration Experiment during Minimal ECF Volume Expansion

\begin{tabular}{|c|c|c|c|c|c|c|c|c|c|c|c|c|}
\hline \multirow{2}{*}{$\begin{array}{l}\text { Clearance } \\
\text { period }\end{array}$} & \multirow[b]{2}{*}{ Time } & \multirow[b]{2}{*}{ GFR } & \multicolumn{3}{|c|}{ Plasma } & \multicolumn{3}{|c|}{ Urine } & \multirow{2}{*}{\multicolumn{2}{|c|}{$\mathrm{HCO}_{3}$ excretion }} & \multirow{2}{*}{\multicolumn{2}{|c|}{$\mathrm{HCO}_{3}$ reabsorption }} \\
\hline & & & $\mathrm{pH}$ & $\mathrm{HCO}_{3}$ & $\mathrm{pCO}_{2}$ & $\mathrm{pH}$ & $\mathrm{HCO}_{3}$ & $\mathrm{pCO}_{2}$ & & & & \\
\hline & $\min$ & $\mathrm{ml} / \mathrm{min}$ & & $\mu E q / m l$ & $m m \mathrm{Hg}$ & & $\mu E q / m l$ & $m m H g$ & $\mu E q / \min$ & $\begin{array}{c}\mu E q / m l \\
G F R\end{array}$ & $\mu E q / \min$ & $\begin{array}{c}\mu E q / m l \\
G F R\end{array}$ \\
\hline & -175 & \multicolumn{11}{|c|}{$\begin{array}{l}\text { Light ether anesthesia for insertion of tail vein and femoral artery cannulae, bladder catheter, and } \\
\text { positioning animal in holder (duration } 25 \mathrm{~min} \text { ) }\end{array}$} \\
\hline & -60 & \multicolumn{11}{|c|}{ Inulin prime $0.71 \mu \mathrm{c}$ inulin- ${ }^{14} \mathrm{C}$ in $1 \mathrm{ml}$ normal saline } \\
\hline & & \multicolumn{11}{|c|}{ Sustaining solution containing $71 \mu \mathrm{c}{ }^{14} \mathrm{C}$ in $100 \mathrm{ml}$ normal saline at $0.11 \mathrm{ml} / \mathrm{min}$} \\
\hline 1 & $0-30$ & 3.09 & 7.41 & 24.0 & 40.0 & 6.70 & 2.83 & 27.0 & 0.46 & 0.15 & 77.4 & 25.1 \\
\hline & $31-49$ & \multicolumn{11}{|c|}{ Sustaining solution containing $\mathrm{NaHCO}_{3} 30 \mathrm{mEq} /$ liter at $0.11 \mathrm{ml} / \mathrm{min}$} \\
\hline 2 & $49-65$ & 2.88 & 7.44 & 24.0 & 36.5 & 6.88 & 3.97 & 24.8 & 0.70 & 0.24 & 71.9 & 25.0 \\
\hline \multirow[t]{2}{*}{3} & $65-77$ & 3.61 & 7.45 & 24.0 & 36.0 & 6.90 & 4.84 & 28.0 & 0.10 & 0.29 & 89.9 & 24.9 \\
\hline & $82-186$ & \multicolumn{11}{|c|}{$\begin{array}{l}\text { Prime } 0.3 \mathrm{ml} 1.5 \mathrm{M} \mathrm{NaHCO} \\
\text { liter at } 0.11 \mathrm{ml} / \mathrm{min}\end{array}$} \\
\hline 4 & $186-200$ & 2.64 & 7.53 & 29.0 & 36.5 & 7.20 & 9.88 & 28.5 & 1.77 & 0.67 & 78.6 & 29.8 \\
\hline \multirow[t]{2}{*}{5} & $200-211$ & 3.61 & 7.54 & 29.5 & 36.0 & 7.25 & 10.4 & 29.5 & 1.89 & 0.52 & 109.8 & 30.5 \\
\hline & $218-238$ & \multicolumn{11}{|c|}{$\begin{array}{l}\text { Prime } 0.3 \mathrm{ml} 1.5 \mathrm{M} \mathrm{NaHCO}\left(0.45 \mathrm{mEq} / \mathrm{HCO}_{3}^{-}\right) \text {, sustaining solution containing } \mathrm{NaHCO}_{3} 40 \\
\mathrm{mEq} / \text { liter at } 0.11 \mathrm{ml} / \mathrm{min}\end{array}$} \\
\hline 6 & $238-250$ & 3.52 & 7.54 & 29.5 & 36.0 & 7.30 & 14.0 & 28.5 & 2.21 & 0.63 & 106.8 & 30.4 \\
\hline \multirow[t]{2}{*}{7} & $250-261$ & 3.27 & 7.53 & 29.7 & 37.0 & 7.30 & 11.2 & 25.0 & 2.03 & 0.62 & 99.9 & 30.6 \\
\hline & $266-289$ & \multicolumn{11}{|c|}{$\begin{array}{l}\text { Prime } 0.3 \mathrm{ml} 1.5 \mathrm{M} \mathrm{NaHCO}_{3}\left(0.45 \mathrm{mEq}^{\mathrm{MCO}_{3}}{ }^{-}\right) \text {, sustaining solution containing } \mathrm{NaHCO}_{3} 80 \\
\mathrm{mEq} / \text { liter at } 0.11 \mathrm{ml} / \mathrm{min}\end{array}$} \\
\hline 8 & $289-317$ & 3.11 & 7.60 & 32.2 & 34.0 & 7.60 & 36.8 & 36.0 & 3.94 & 1.27 & 101.6 & 32.7 \\
\hline \multirow[t]{2}{*}{9} & $317-330$ & 3.78 & 7.59 & 33.1 & 35.8 & 7.60 & 36.3 & 38.0 & 4.46 & 1.18 & 126.9 & 33.6 \\
\hline & $334-350$ & \multicolumn{11}{|c|}{$\begin{array}{l}\text { Prime } 0.4 \mathrm{ml} 1.5 \mathrm{M} \mathrm{NaHCO}\left(0.60 \mathrm{mEq} \mathrm{HCO}_{3}\right) \text {, sustaining solution containing } \mathrm{NaHCO}_{3} 100 \\
\mathrm{mEq} / \text { liter at } 0.11 \mathrm{ml} / \mathrm{min}\end{array}$} \\
\hline 10 & $350-362$ & 2.60 & 7.61 & 39.0 & 40.5 & 7.85 & 84.7 & 45.5 & 5.68 & 2.18 & 100.8 & 38.8 \\
\hline \multirow[t]{2}{*}{11} & $362-374$ & 3.18 & 7.59 & 38.7 & 41.0 & 7.85 & 80.0 & 45.0 & 8.64 & 2.72 & 120.6 & 37.9 \\
\hline & $379-392$ & \multicolumn{11}{|c|}{$\begin{array}{l}\text { Prime } 0.4 \mathrm{ml} 1.5 \mathrm{M} \mathrm{NaHCO}_{3}\left(0.60{\mathrm{mEq} \mathrm{HCO}_{3}}^{-}\right) \text {, sustaining solution containing } \mathrm{NaHCO}_{3} 100 \\
\mathrm{mEq} / \text { liter at } 0.11 \mathrm{ml} / \mathrm{min}\end{array}$} \\
\hline 12 & $392-403$ & 2.99 & 7.61 & 40.8 & 41.5 & 7.95 & 117.2 & 50.0 & 8.56 & 2.86 & 119.5 & 40.0 \\
\hline \multirow[t]{2}{*}{13} & $403-416$ & 2.55 & 7.60 & 39.0 & 41.3 & 7.85 & 72.9 & 41.0 & 5.90 & 2.31 & 98.5 & 38.7 \\
\hline & $421-436$ & \multicolumn{11}{|c|}{$\begin{array}{l}\text { Prime } 0.4 \mathrm{ml} \mathrm{M} \mathrm{NaHCO} \\
\text { liter } 0.11 \mathrm{ml} / \mathrm{min}\end{array}$} \\
\hline 14 & $436-447$ & 3.16 & 7.62 & 43.0 & 43.3 & 7.85 & 82.9 & 44.5 & 6.63 & 2.10 & 136.0 & 43.1 \\
\hline \multirow[t]{2}{*}{15} & $447-461$ & 2.78 & 7.62 & 43.0 & 43.3 & 7.90 & 82.9 & 42.5 & 8.29 & 2.98 & 117.2 & 42.2 \\
\hline & $466-491$ & $\begin{array}{r}\text { Prime } \\
\mathrm{mE}\end{array}$ & $\begin{array}{l}0.4 \mathrm{ml} \\
/ \text { liter a }\end{array}$ & $\begin{array}{l}.5 \mathrm{M} \mathrm{Na} \\
0.11 \mathrm{ml}\end{array}$ & $\begin{array}{l}\mathrm{HCO}_{3}( \\
/ \mathrm{min}\end{array}$ & $60 \mathrm{mE}$ & $\left.\mathrm{HCO}_{3}^{-}\right)$ & ustainin & solution & containi & ng $\mathrm{NaHC}$ & $\mathrm{O}_{3} 120$ \\
\hline 16 & $491-502$ & 3.12 & 7.65 & 47.0 & 45.5 & 7.75 & 75.9 & 55.0 & 8.28 & 2.65 & 145.7 & 46.7 \\
\hline 17 & $502-523$ & 2.58 & 7.64 & 45.6 & 45.5 & 7.75 & 69.6 & 54.0 & 8.63 & 3.34 & 114.9 & 44.5 \\
\hline & $526-549$ & $\begin{array}{r}\text { Prime } \\
\mathrm{mE}\end{array}$ & $\begin{array}{l}.4 \mathrm{ml} \\
\text { /liter a }\end{array}$ & $\begin{array}{l}.5 \mathrm{M} \mathrm{Na} \\
0.11 \mathrm{~m}\end{array}$ & $\begin{array}{l}\mathrm{HCO}_{3}( \\
/ \mathrm{min}\end{array}$ & $.60 \mathrm{mE}$ & $\left.\mathrm{HCO}_{3}\right)$, & ustainin & solution & containi & ng $\mathrm{NaHC}$ & $\mathrm{O}_{3} 120$ \\
\hline 18 & 549-564 & 2.46 & 7.65 & 49.2 & 46.8 & 7.80 & 113.5 & 70.0 & 11.4 & 4.61 & 115.8 & 47.0 \\
\hline 19 & $564-575$ & 2.57 & 7.65 & 49.7 & 46.5 & 7.90 & 94.6 & 52.0 & 13.7 & 5.34 & 120.4 & 46.9 \\
\hline & $577-601$ & $\begin{array}{l}\text { Prime } \\
\mathrm{mE}\end{array}$ & $\begin{array}{l}.4 \mathrm{ml} \\
\text { /liter }\end{array}$ & $\begin{array}{l}.5 \mathrm{M} \mathrm{Na} \\
0.11 \mathrm{~m}\end{array}$ & $\begin{array}{l}\mathrm{HCO}_{3}( \\
/ \mathrm{min}\end{array}$ & $50 \mathrm{mE}$ & $\left.\mathrm{HCO}_{3}^{-}\right)$, & sustainin & g solution & containi & ng NaHC & $\mathrm{O}_{3} 120$ \\
\hline 20 & $601-620$ & 2.59 & 7.71 & 57.7 & 47.8 & 7.80 & 132.8 & 80.0 & 13.3 & 5.13 & 143.6 & 55.5 \\
\hline 21 & $620-637$ & 2.49 & 7.68 & 57.5 & 50.0 & 7.80 & 65.8 & 43.5 & 8.16 & 3.28 & 142.2 & 57.1 \\
\hline
\end{tabular}

Rat weight, $220 \mathrm{~g}$. 


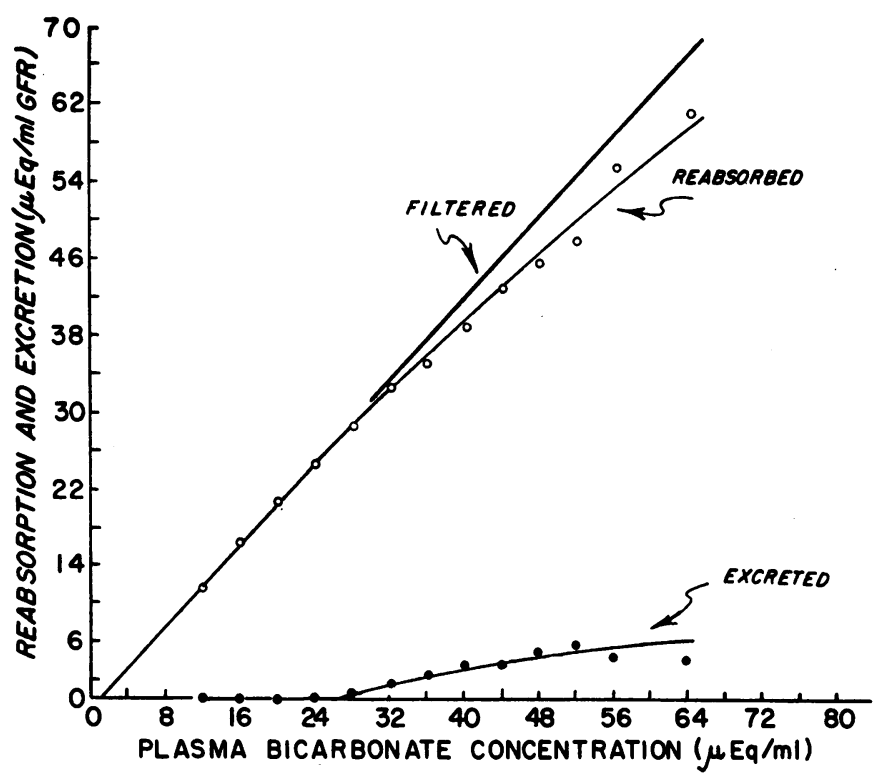

FIGURE 1 Mean bicarbonate titration curves for nine animals studied under conditions of minimized ECF volume expansion.

to $120 \mathrm{mEq} /$ liter. The concentration of chloride, the only other anion, was changed reciprocally. 15-20 clearance periods, each 10-30 min in duration, were obtained. Observations were made over a range of plasma bicarbonate concentrations extending from values as low as $11 \mathrm{mEq} / \mathrm{liter}$ to values as high as $64 \mathrm{mEq} / \mathrm{liter}$. Two to three clearance periods were obtained at each level of bicarbonate infusion. In approximately half of the experiments $2.5 \%$ ammonium chloride was administered in the drinking water the night before study to effect a decrease in the initial plasma bicarbonate concentrations to subnormal levels.

Glomerular filtration rate was measured using carboxyllabeled inulin $-{ }^{14} \mathrm{C}$. A priming dose of $0.7 \mu \mathrm{c}$ of inulin $-{ }^{14} \mathrm{C}$ in $1 \mathrm{ml}$ of isotonic saline was administered intravenously. Sufficient inulin $-{ }^{14} \mathrm{C}$ was added to the sustaining solutions to provide counting rates at least 10 times greater than background in $10-\mu 1$ samples of plasma.

For experiments in which ECF volume expansion was minimized, the sustaining solutions containing $\mathrm{NaHCO}_{3}$, $\mathrm{NaCl}$, and inulin were infused at a rate of 0.09 or 0.11 $\mathrm{ml} / \mathrm{min}$. Before each increment in the rate of bicarbonate infusion, a single injection of 0.4 or $0.6 \mathrm{mEq}$ of $\mathrm{HCO}_{3}$ was infused in a volume of 0.3 or $0.4 \mathrm{ml}$. An equilibration period of at least $12 \mathrm{~min}$ was allowed after initiating each new sustaining solution. Exaggerated extracellular fluid volume expansion was accomplished as follows: after obtaining two control clearance periods, isotonic sodium chloride containing appropriate concentrations of inulin $-{ }^{14} \mathrm{C}$ was infused at 0.15 $\mathrm{ml} / \mathrm{min}$ for $20 \mathrm{~min}, 0.375 \mathrm{ml} / \mathrm{min}$ for the next $20 \mathrm{~min}$, and $0.75 \mathrm{ml} / \mathrm{min}$ for the following $20 \mathrm{~min}$. The infusion then was continued at $0.46 \mathrm{ml} / \mathrm{min}$ and two new control clearance periods were obtained. At this point, a bicarbonate-containing solution was substituted for the sodium chloride and the titration studies were performed using a pattern of increasing bicarbonate concentrations in the infusate similar to that described in the first group of experiments. The infusion rate for these experiments, however, was maintained at 0.46 $\mathrm{ml} / \mathrm{min}$ instead of $0.1 \mathrm{ml} / \mathrm{min}$.
All urine samples were collected under oil, and blood samples were obtained directly from the indwelling femoral arterial cannula. The $\mathrm{pH}$ and $\mathrm{pCO}_{2}$ determinations were made immediately after collection of blood and urine using an Instrumentation Laboratory, Inc. microgas analyzer (Model IL 113-FL). Bicarbonate concentrations in urine and plasma were calculated using the Henderson-Hasselbalch equation with a $\mathrm{pk}^{\prime}$ value of 6.1 and an $\alpha$ value of 0.0301 for plasma. An $\alpha$ value of 0.0309 was used for urine and $\mathrm{pk}^{\prime}$ values were calculated for each urine sample using the formula $\mathrm{pk}^{\prime}=6.33$ $-0.5 \times \sqrt{\mathrm{B}}$, where $\mathrm{B}$ represents the total cation concentration estimated as the sum of sodium concentration plus

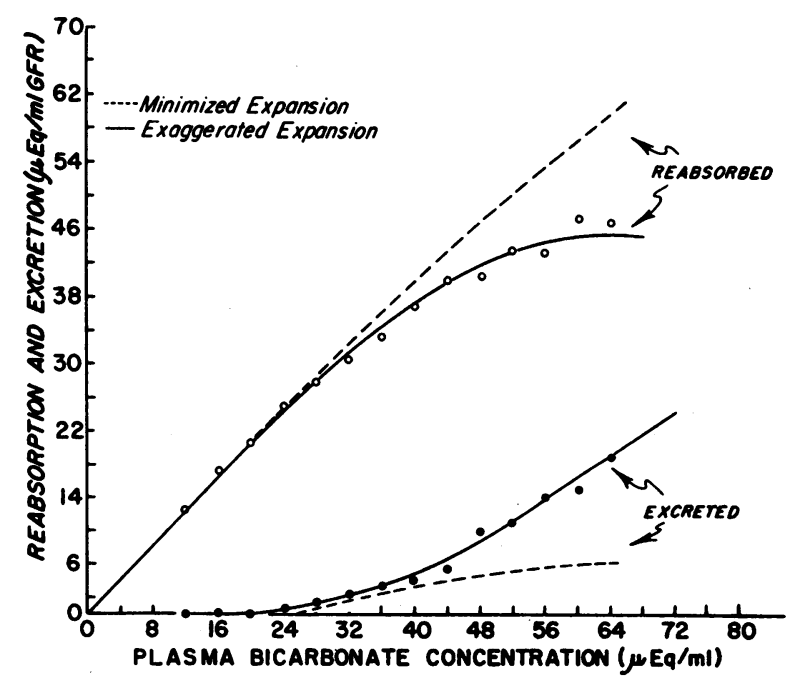

FIgURE 2 Mean bicarbonate titration curves for six animals studied under conditions of exaggerated ECF volume expansion. 
potassium concentration. Inulin- ${ }^{14} \mathrm{C}$ in plasma and urine samples was counted in a Packard Tri-Carb liquid scintillation counter (Model 3214); at least 10,000 counts were obtained in all urine and plasma samples. Bicarbonate reabsorption was calculated as the difference between the amount filtered and the amount excreted. A Donnan factor of 1.05 was employed for estimating the concentration of bicarbonate in the ultrafiltrate. Sodium was determined using an Instrumentation Laboratories flame photometer.

\section{RESULTS}

The results of a representative bicarbonate titration study in an animal in which extracellular fluid volume expansion was minimized are shown in Table I. The plasma bicarbonate concentration was increased from an initial level of $24 \mathrm{mEq} /$ liter to a final value of $58 \mathrm{mEq} /$ liter. The arterial $\mathrm{pCO}_{2}$ was $40 \mathrm{~mm} \mathrm{Hg}$ in the control period; it then decreased to $36 \mathrm{~mm} \mathrm{Hg}$ and rose pro-

TABLE II

Representative Bicarbonate Titration Experiment during Exaggerated ECF Volume Expansion

\begin{tabular}{|c|c|c|c|c|c|c|c|c|c|c|c|c|}
\hline \multirow{2}{*}{$\begin{array}{l}\text { Clearance } \\
\text { period }\end{array}$} & \multirow[b]{2}{*}{ Time } & \multirow[b]{2}{*}{ GFR } & \multicolumn{3}{|c|}{ Plasma } & \multicolumn{3}{|c|}{ Urine } & \multirow{2}{*}{\multicolumn{2}{|c|}{$\mathrm{HCO}_{3}$ excretion }} & \multirow{2}{*}{\multicolumn{2}{|c|}{$\mathrm{HCO}_{3}$ reabsorption }} \\
\hline & & & $\mathrm{pH}$ & $\mathrm{HCO}_{3}$ & $\mathrm{pCO}_{2}$ & $\mathrm{pH}$ & $\mathrm{HCO}_{3}$ & $\mathrm{pCO}_{2}$ & & & & \\
\hline & $\min$ & $m l / m i n$ & & $\mu E q / m l$ & $m m \mathrm{Hg}$ & & $\mu E q / m l$ & $m m \mathrm{Hg}$ & $\mu E q / \min$ & $\begin{array}{c}\mu E q / m l \\
G F R\end{array}$ & $\mu E q / \min$ & $\begin{array}{l}\mu E q / m l \\
G F R\end{array}$ \\
\hline & -187 & \multicolumn{11}{|c|}{$\begin{array}{l}\text { Light ether anesthesia for insertion of tail vein and femoral artery cannulae and bladder catheter, } \\
\text { and positioning animal in holder (duration } 40 \mathrm{~min} \text { ) }\end{array}$} \\
\hline & -62 & \multicolumn{11}{|c|}{$\begin{array}{l}\text { Inulin prime } 0.7 \mu \mathrm{c} \text { inulin }-{ }^{14} \mathrm{C} \text { in } 1 \mathrm{ml} \text { normal saline, i.v. Sustaining solution begun containing } 71 \\
\mu \mathrm{c} \text { inulin }{ }^{-14} \mathrm{C} \text { in } 100 \mathrm{ml} \text { normal saline }\end{array}$} \\
\hline 1 & $0-23$ & 1.65 & 7.37 & 18.8 & 33.5 & 5.80 & 0.30 & 20.0 & 0.01 & 0.01 & 32.5 & 19.7 \\
\hline \multirow[t]{5}{*}{2} & $23-43$ & 1.99 & 7.37 & 19.1 & 34.0 & 5.78 & 0.29 & 21.0 & 0.01 & 0.01 & 39.8 & 20.0 \\
\hline & $43-63$ & \multicolumn{11}{|c|}{ Normal saline containing inulin $-{ }^{14} \mathrm{C}(17 \mu \mathrm{c}$ in $100 \mathrm{ml}$ normal saline $)$ at $0.15 \mathrm{ml} / \mathrm{min}$} \\
\hline & $63-83$ & \multicolumn{11}{|c|}{ Normal saline containing inulin ${ }^{-14} \mathrm{C}(17 \mu \mathrm{c}$ in $100 \mathrm{ml}$ normal saline $)$ at $0.375 \mathrm{ml} / \mathrm{min}$} \\
\hline & $83-103$ & \multicolumn{11}{|c|}{ Normal saline containing inulin $-{ }^{14} \mathrm{C}(17 \mu \mathrm{c}$ in $100 \mathrm{ml}$ normal saline $)$ at $0.75 \mathrm{ml} / \mathrm{min}$} \\
\hline & $103-125$ & \multicolumn{11}{|c|}{ Normal saline containing inulin $-{ }^{14} \mathrm{C}(17 \mu \mathrm{c}$ in $100 \mathrm{ml}$ normal saline $)$ at $0.46 \mathrm{ml} / \mathrm{min}$} \\
\hline 3 & $125-133$ & 2.88 & 7.34 & 19.5 & 37.5 & 6.00 & 0.57 & 25.0 & 0.32 & 0.11 & 58.7 & 20.3 \\
\hline \multirow[t]{2}{*}{4} & $133-143$ & 2.56 & 7.35 & 19.8 & 37.5 & 6.00 & 0.55 & 24.0 & 0.25 & 0.10 & 53.0 & 20.6 \\
\hline & $148-168$ & \multicolumn{11}{|c|}{$\begin{array}{l}\text { Sustaining solution with } 17 \mu \mathrm{c} \text { inulin- }-{ }^{14} \mathrm{C} / 100 \mathrm{ml} \text { and } \mathrm{NaHCO}_{3} 30 \mathrm{mEq} / \text { liter solution, infusion } \\
\text { rate } 0.46 \mathrm{ml} / \mathrm{min}\end{array}$} \\
\hline 5 & $168-178$ & 2.78 & 7.42 & 23.5 & 37.5 & 6.12 & 0.80 & 26.5 & 0.32 & 0.12 & 68.3 & 24.6 \\
\hline \multirow[t]{2}{*}{6} & $178-189$ & 2.72 & 7.45 & 25.6 & 37.5 & 6.03 & 0.70 & 29.3 & 0.29 & 0.11 & 72.8 & 26.8 \\
\hline & $195-210$ & \multicolumn{11}{|c|}{$\begin{array}{l}\text { Prime } 0.3 \mathrm{ml} 1.5 \mathrm{~m} \mathrm{NaHCO} \text { (in D. W. }\left(0.45 \mathrm{mEq} \mathrm{HCO}_{3}^{-}\right) \text {, sustaining solution containing } \\
\mathrm{NaHCO}_{3} 30 \mathrm{mEq} / \text { liter, infusion rate } 0.46 \mathrm{ml} / \mathrm{min}\end{array}$} \\
\hline 7 & $210 / 218$ & 2.89 & 7.55 & 34.7 & 40.8 & 6.95 & 6.58 & 31.5 & 2.30 & 0.70 & 103.0 & 35.6 \\
\hline \multirow[t]{2}{*}{8} & $218-228$ & 3.05 & 7.56 & 35.3 & 41.0 & 7.00 & 8.06 & 36.0 & 3.71 & 1.21 & 109.3 & 35.7 \\
\hline & $233-248$ & \multicolumn{11}{|c|}{$\begin{array}{l}\text { Sustaining solution with } 17 \mu \mathrm{c} \text { inulin- }{ }^{14} \mathrm{C} / 100 \mathrm{ml} \text { and } \mathrm{NaHCO}_{3} 40 \mathrm{mEq} / \text { liter, infustion rate } \\
0.46 \mathrm{ml} / \mathrm{min}\end{array}$} \\
\hline 9 & $248-258$ & 2.99 & 7.61 & 39.0 & 40.5 & 7.10 & 9.64 & 35.0 & 4.34 & 1.45 & 118.1 & 39.5 \\
\hline \multirow[t]{2}{*}{10} & $258-273$ & 2.39 & 7.62 & 38.3 & 39.0 & 7.24 & 16.1 & 41.5 & 6.29 & 2.63 & 89.8 & 37.6 \\
\hline & $277-287$ & \multicolumn{11}{|c|}{$\begin{array}{l}\text { Sustaining solution with } 17 \mu \mathrm{c} \text { inulin- }{ }^{-14} \mathrm{C} / 100 \mathrm{ml} \text { and } \mathrm{NaHCO}_{3} 60 \mathrm{mEq} / \text { liter, infusion rate } \\
0.46 \mathrm{ml} / \mathrm{min}\end{array}$} \\
\hline 11 & $287-297$ & 1.97 & 7.64 & 42.3 & 40.8 & 7.42 & 22.4 & 38.0 & 6.71 & 3.39 & 80.8 & 40.8 \\
\hline \multirow[t]{2}{*}{12} & $297-308$ & 1.68 & 7.65 & 43.2 & 40.8 & 7.50 & 23.9 & 34.5 & 6.44 & 3.38 & 69.8 & 41.5 \\
\hline & $312-322$ & \multicolumn{11}{|c|}{$\begin{array}{l}\text { Prime } 0.3 \mathrm{ml} 1.5 \mathrm{M} \mathrm{NaHCO} \text { (in D. W.) }\left(0.45 \mathrm{mEq} \mathrm{HCO}_{3}^{-}\right) \text {, sustaining solution containing } \\
\mathrm{NaHCO}_{3} 60 \mathrm{mEq} / \text { liter, infusion rate } 0.46 \mathrm{ml} / \mathrm{min}\end{array}$} \\
\hline 13 & $322-334$ & 2.50 & 7.67 & 45.8 & 41.5 & 7.60 & 41.5 & 43.5 & 14.1 & 5.65 & 106.1 & 42.4 \\
\hline \multirow[t]{2}{*}{14} & $334-344$ & 2.25 & 7.66 & 46.2 & 42.5 & 7.64 & 43.5 & 43.5 & 15.2 & 6.77 & 93.9 & 41.7 \\
\hline & $344-357$ & \multicolumn{11}{|c|}{$\begin{array}{l}\text { Prime } 0.3 \mathrm{ml} 1.5 \mathrm{M} \mathrm{NaHCO} \text { (in D. W.) }\left(0.45 \mathrm{mEq}^{\mathrm{NCO}}{ }_{3}^{-}\right) \text {, sustaining solution containing } \\
\mathrm{NaHCO}_{3} 80 \mathrm{mEq} / \text { liter, infusion rate } 0.46 \mathrm{ml} / \mathrm{min}\end{array}$} \\
\hline 15 & $357-375$ & 2.73 & 7.70 & 49.0 & 42.0 & 7.62 & 49.5 & 47.2 & 16.3 & 5.98 & 124.1 & 45.5 \\
\hline 16 & $375-387$ & 1.96 & 7.69 & 51.5 & 44.5 & 7.65 & 57.3 & 53.5 & 18.9 & 9.65 & 87.1 & 44.4 \\
\hline & $392-402$ & $\begin{array}{l}\text { Prime } \\
\mathrm{NaH}\end{array}$ & $\begin{array}{l}0.3 \mathrm{ml} \\
\mathrm{CO}_{3} 80\end{array}$ & $\begin{array}{l}.5 \mathrm{M} \mathrm{Na} \\
\mathrm{nEq} / \mathrm{lite}\end{array}$ & $\begin{array}{l}\mathrm{HCO}_{3}(\mathrm{i} \\
\text {, infusio }\end{array}$ & $\begin{array}{l}\text { D. Y } \\
\text { rate } 0\end{array}$ & $(0.45$ & $\mathrm{Eq} \mathrm{HCC}$ & $\left.3^{-}\right)$, susta & ining so & ution cont & taining \\
\hline 17 & $402-413$ & 1.41 & 7.69 & 54.5 & 46.0 & 7.71 & 69.2 & 55.0 & 20.8 & 14.7 & 59.9 & 42.5 \\
\hline 18 & $413-426$ & 1.92 & 7.68 & 52.5 & 46.0 & 7.80 & 83.7 & 54.0 & 29.3 & 15.3 & 76.6 & 39.9 \\
\hline
\end{tabular}

Rat weight, $190 \mathrm{~g}$; 
TABLE III

Sodium and Bicarbonate Excretion

\begin{tabular}{|c|c|c|c|c|}
\hline & \multirow[b]{2}{*}{$\mathrm{C}_{\mathrm{In}}$} & \multicolumn{3}{|c|}{ Plasma } \\
\hline & & $\mathrm{HCO}_{3}$ & $\mathrm{pH}$ & $\mathrm{pCO}_{2}$ \\
\hline & $\mathrm{ml} / \mathrm{min}$ & $\mu E q / m l$ & & $m m \mathrm{Hg}$ \\
\hline \multicolumn{5}{|l|}{ Control } \\
\hline $\begin{array}{l}\text { "Minimal" expansion }(n=9) \\
\text { "Exaggerated" expansion }(n=6)\end{array}$ & $2.78 \pm 0.19$ & $19.7 \pm 1.96$ & $7.38 \pm 0.03$ & $33.5 \pm 1.81$ \\
\hline Before expansion & $2.27 \pm 0.14$ & $20.4 \pm 2.04$ & $7.36 \pm 0.03$ & $36.6 \pm 2.46$ \\
\hline After expansion & $2.87 \pm 0.14$ & $19.6 \pm 1.64$ & $7.34 \pm 0.03$ & $37.2 \pm 1.37$ \\
\hline \multicolumn{5}{|l|}{ Bicarbonate diuresis } \\
\hline “Minimal” expansion & $2.47 \pm 0.21$ & $46.1 \pm 1.86$ & $7.66 \pm 0.01$ & $42.1 \pm 1.04$ \\
\hline "Exaggerated" expansion & $2.73 \pm 0.03$ & $47.5 \pm 2.84$ & $7.67 \pm 0.02$ & $42.9 \pm 1.23$ \\
\hline
\end{tabular}

Values represent means $\pm \mathrm{SE}$ of means. Values during control conditions were selected from a compilation of one or more control clearance periods from each rat under the specified conditions. Values during bicarbonate diuresis were derived by selecting clearance periods in which plasma bicarbonate concentrations in the minimal and exaggerated expansion groups were comparable and then recording the values for plasma $\mathrm{pH}$ and $\mathrm{pCO}_{2}$ urine $\mathrm{pH}, \mathrm{pCO}_{2}$, bicarbonate excretion, and sodium excretion.

gressively thereafter to a final value of 50 . Bicarbonate reabsorption increased over the entire range of plasma bicarbonate concentrations and no $\mathrm{Tm}$ or tendency for a $\mathrm{Tm}$ was observed even at the highest plasma level achieved. Thus with a plasma bicarbonate concentration of $57.5 \mathrm{mEq} /$ liter, bicarbonate reabsorption was 57.1 $\mu \mathrm{Eq} / \mathrm{ml}$ GFR. Bicarbonate excretion increased gradually with the increments in the plasma bicarbonate concentrations but the highest rate of excretion was $13.7 \mu \mathrm{Eq} / \mathrm{min}$ with a filtered load of $134 \mu \mathrm{Eq} / \mathrm{min}$.

Fig. 1 depicts a composite titration curve for nine animals in which ECF volume expansion was minimized. Each point is the mean of from 2 to 25 individual observations. Consistent with the result shown in Table I, mean bicarbonate reabsorption increased progressively over a range of plasma bicarbonate concentrations extending from 12 to $66 \mathrm{mEq} /$ liter. No Tm was demonstrable for the group data despite multiple observations at plasma levels over $45 \mathrm{mEq} /$ liter. Bicarbonate excretion did not begin until plasma bicarbonate concentrations exceeded $27 \mathrm{mEq} /$ liter.

A representative titration study in which extracellular fluid volume expansion was exaggerated is shown in Table II. Plasma bicarbonate concentrations increased from 19 to $54.5 \mathrm{mEq} /$ liter. $\mathrm{pCO}_{2}$ values rose from 33.5 to $46 \mathrm{~mm} / \mathrm{Hg}$. In contrast to the pattern presented in Table I and pictured in Fig. 1, a clearly discernible tendency towards stabilization of bicarbonate reabsorption is evident with a Tm value between 40 and $45 \mu \mathrm{Eq} / \mathrm{ml}$ GFR. In the final portion of the experiment, bicarbonate excretion approximated $15 \mu \mathrm{Eq} / \mathrm{ml}$ GFR in contrast to the maximal value of $5.3 \mu \mathrm{Eq} / \mathrm{ml}$ GFR shown in the representative experiment in Table I for minimized expansion. A composite titration curve for six rats studied during exaggerated extracellular fluid volume expansion is shown in Fig. 2. The pattern for the animals

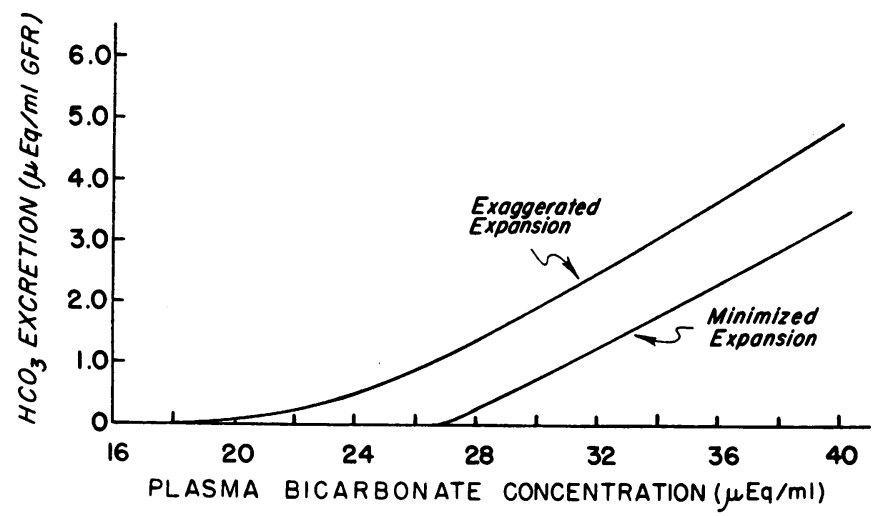

Figure 3 Bicarbonate excretion for animals with minimized and exaggerated ECF volume expansion. 


\begin{tabular}{|c|c|c|c|c|c|}
\hline \multicolumn{6}{|c|}{ Urine } \\
\hline $\mathrm{pH}$ & $\mathrm{pCO}_{2}$ & $\mathrm{UHCO}^{\mathrm{V}} \mathrm{V}$ & $\frac{\mathrm{UHCO}_{\mathrm{HC}} \mathrm{V}}{\mathrm{GFR}}$ & $\mathrm{Na}$ & $\begin{array}{c}\text { Filtered } \mathrm{Na}^{+} \\
\text {excreted }\end{array}$ \\
\hline & $m m \mathrm{Hg}$ & $\mu E q / \min$ & & $\mu E q / \min$ & $\%$ \\
\hline $6.31 \pm 0.17$ & $22.3 \pm 1.61$ & $0.11 \pm 0.49$ & $0.04 \pm 0.05$ & $8.0 \pm 2.32$ & $1.92 \pm 0.49$ \\
\hline $6.28 \pm 0.24$ & $21.8 \pm 1.70$ & $0.15 \pm 0.08$ & $0.06 \pm 0.03$ & $10.4 \pm 2.10$ & $3.37 \pm 0.75$ \\
\hline $6.23 \pm 0.12$ & $25.4 \pm 1.44$ & $0.56 \pm 0.14$ & $0.20 \pm 0.05$ & $71.3 \pm 4.65$ & $17.82 \pm 1.44$ \\
\hline $8.01 \pm 0.04$ & $50.9 \pm 5.64$ & $11.34 \pm 1.68$ & $4.75 \pm 0.76$ & $19.7 \pm 2.43$ & $6.32 \pm 0.90$ \\
\hline $7.69 \pm 0.05$ & $55.5+3.79$ & $27.70+3.97$ & $10.19 \pm 1.56$ & $78.2 \pm 7.41$ & $20.76 \pm 2.58$ \\
\hline
\end{tabular}

with minimized expansion is superimposed for comparison. In the animals with exaggerated expansion, reabsorption tended to stabilize above a plasma bicarbonate concentration of $45 \mu \mathrm{Eq} / \mathrm{ml}$ and there is an apparent Tm for bicarbonate reabsorption with a value of approximately $46 \mu \mathrm{Eq} / \mathrm{ml}$ GFR. Bicarbonate excretion began earlier in the animals with exaggerated expansion than in those with minimized expansion and slope of the excretion curve is much steeper at higher plasma bicarbonate levels.

In Fig. 3, urinary excretion of bicarbonate at increasing plasma bicarbonate concentrations is compared in the animals with minimized and exaggerated expansion of ECF volume. Bicarbonate excretion began at a lower plasma level in the more expanded group and at all levels of plasma bicarbonate above the respective thresholds, excretion rates were greater in animals with exaggerated expansion than in those with minimized expansion. Table III presents comparative data for both groups of animals for plasma $\mathrm{pH}, \mathrm{pCO}_{2}$, fractional sodium excretion, and certain other relevant parameters during the control periods, and after brisk bicarbonate excretion was in effect. At the same plasma bicarbonate concentration, bicarbonate excretion per unit of GFR was approximately twice as great in the animals with exaggerated expansion. However no differences in either $\mathrm{pH}$ or $\mathrm{pCO}_{2}$ were evident between the two groups. The patterns of sodium excretion, on the other hand, were markedly different with the exaggerated expansion group excreting $21 \%$ of the filtered sodium while the minimal expansion group excreted only $6.0 \%$. The absolute rates of sodium excretion (in $\mu \mathrm{Eq} / \mathrm{min}$ ) were 78.2 and 19.7 respectively; only about $25 \%$ of this difference could be attributed to bicarbonate as an impermeant anion.

\section{DISCUSSION}

The standard procedure for examining bicarbonate reabsorption consists of infusing bicarbonate solutions so as to effect a gradual but progressive increment in plasma bicarbonate concentrations. Since bicarbonate is infused as a sodium salt, large quantities of sodium are administered during the course of a classical titration experiment. Hence, expansion of the extracellular fluid volume is an inescapable consequence of the experimental method. There is now compelling evidence that ECF volume expansion leads to striking alterations of proximal tubular functions. The best characterized of these is the inhibition of fractional sodium reabsorption $(4,5)$. However glucose reabsorption is altered (6) and in the dog, maximum tubular absorption rate for $p$-aminohippuric acid (TmPar) is diminished. ${ }^{1}$ There are also observations that suggest that calcium (7), magnesium (7), and urate reabsorption (8) may be influenced by ECF expansion. The reabsorption of bicarbonate in the proximal tubule presumably is coupled to sodium reabsorption either directly or indirectly whether this reabsorption occurs in consequence of the secretion of hydrogen ions into the tubular lumen or the transport of bicarbonate as an ion. Thus the possibility exists that the pattern of bicarbonate reabsorption which has been accepted as normal may in fact represent an altered pattern which conceals substantial inhibition of bicarbonate reabsorptive capacity. In micropuncture studies, recently described by Kunau, Frick, Rector, and Seldin (9) estimated tubular fluid/plasma ratios for bicarbonate (estimated from tubular fluid/plasma chloride ratios) in su-

${ }^{1}$ Shapiro, H., M. Lao, C. Manley, R. G. Schultze, and N. S. Bricker. Unpublished observations. 
perficial nephrons of rats were substantially higher during saline loading than in the hydropenic state. These data indicate that ECF volume does inhibit bicarbonate reabsorption in the proximal tubule. This inhibition could affect not only the apparent $T \mathrm{~m}$ for bicarbonate but also the threshold level at which bicarbonate first appears in the urine.

The present studies support the foregoing possibility. When ECF volume expansion was minimized during the execution of titration experiments, no $\mathrm{Tm}$ was demonstrated in the majority of individual rats studied or in a group plot from nine experiments despite the fact that plasma bicarbonate concentrations were elevated to values in excess of $60 \mathrm{mEq}$ /liter. Conversely, when extracellular fluid volume expansion was exaggerated, a clear Tm could be demonstrated and the threshold for bicarbonate excretion was diminished. The data, therefore, suggest that the titration procedure as typically employed leads to inhibition of proximal tubular reabsorption of bicarbonate; thus the apparent $\mathrm{Tm}$ observed in such conditions is not a true index of the maximum capacity for proximal tubular reabsorption of bicarbonate. Why the values for bicarbonate reabsorption were so high even in the expanded rats is not evident, but this presumably relates to a species difference between the rat and other species previously studied (in man and dogs).

\section{ACKNOWLEDGMENT}

This work was supported by U. S. Public Health Service Research Grant No. AM-09976.

\section{REFERENCES}

1. Pitts, R. F., and W. D. Lotspeich. 1946. Bicarbonate and the renal regulation of acid base balance. Amer. J. Physiol. $147: 138$.

2. Pitts, R. F., J. L. Ayer, and W. A. Schiess. 1949. The renal regulation of acid-base balance in man. III. The reabsorption and excretion of bicarbonate. J. Clin. Invest. 28: 35 .

3. Shankel, S. W., A. M. Robson, and N. S. Bricker. 1967. On the mechanism of the splay in the glucose titration curve in advanced experimental renal disease in the rat. J. Clin. Invest. 46: 164.

4. Dirks, J. H., W. J. Cirksena, and R. W. Berliner. 1965. The effect of saline infusion on sodium reabsorption by the proximal tubule of the dog. J. Clin. Invest. 44: 1160 .

5. Cortney, M. A., M. Mylle, W. E. Lassiter, and C. W. Gottschalk. 1965. Renal tubular transport of water, solute, and $\mathrm{PAH}$ in rats loaded with isotonic saline. Amer. J. Physiol. 209: 1199.

6. Robson, A. M., P. L. Srivastava, and N. S. Bricker. 1968. The influence of saline loading on renal glucose reabsorption in the rat. J. Clin. Invest. 47: 329.

7. Massry, S. G., J. W. Coburn, L. W. Chapman, and C. R. Kleeman. 1967. Effect of $\mathrm{NaCl}$ infusion on urinary $\mathrm{Ca}^{++}$ and $\mathrm{Mg}^{++}$during reduction in their filtered loads. Amer. J. Physiol. 213: 1218.

8. Steele, T. H. 1968. The latent effect of volume expansion on urate reabsorption in man. Clin. Res. 16: 397. (Abstr.)

9. Kunau, R., A. Frick, F. C. Rector, Jr., and D. W. Seldin. 1966. Effect of extracellular fluid (ECF) volume expansion, $\mathrm{K}^{+}$deficiency and $\mathrm{pCO}_{2}$ on bicarbonate reabsorption in the rat kidney. Clin. Res. 14: 380. (Abstr.) 